\title{
Estrategias de mercado para la exportación de arandano (Vaccinium corymbosum L.), de la
} región Ica

\author{
Market strategies for the export of blueberry (Vaccinium corymbosum L.), from the Ica region
}

\author{
Antonina Juana García Espinoza \\ antonina.garcia@ unica.edu.pe - Universidad Nacional San Luis Gonzaga, Ica \\ Dante Fermín Calderón Huamani \\ dante.calderon@ unica.edu.pe - Universidad Nacional San Luis Gonzaga, Ica \\ Domingo Jesús Cabel Moscoso \\ dcabel@ unaj.edu.pe - Universidad Nacional de J uliaca, J uliaca \\ Cedidec García Espinoza \\ cedidec.garcia.espinoza@gmail.com - Universidad Nacional San Luis Gonzaga, Ica
}

Recibido el 18/09/20 | Aceptado el 02/10/20

DOI: https://doi.org/10.47190/nric.v3i1.129

\section{Resumen}

El fruto de arándano es una excelente fuente de vitamina C. Asimismo, existen segmentos de mercado potencial en Estados Unidos que demanda esta fruta. Por lo que, la presente investigación titulada: ESTRATEGIAS DE MERCADO PARA LA EXPORTACIÓN DE ARÁNDANOS (Vaccinium spp.), DE LA REGION ICA, plantea el problema de investigación: ¿Cuál es la relación que existe en la aplicación de estrategias de mercado y el incremento de las exportaciones de Arándanos frescos (Vaccinium spp.) de la Región Ica? y tiene como objetivo: Determinar la relación que existe en la aplicación de estrategias de mercado y el incremento de las exportaciones de Arándanos frescos (Vaccinium spp.) de la región Ica. Se partió de la hipótesis general: La aplicación de estrategias de mercado tiene relación con el incremento de las exportaciones de Arándanos frescos (Vaccinium spp.) de la Región Ica. Se realizó una revisión inicial de la descripción biológica del fruto, de la calidad, de las exportaciones, precios, comercialización y principales empresas nacionales e internaciones de producción de esta fruta. Asimismo, se realizó un análisis de las diferentes estrategias de mercado y se determinó que las estrategias de mercado que deben ser implementadas por las empresas de la región de Ica son: Estrategias de marketing de comunicación y promoción, estrategia del producto y estrategia de diversificación concéntrica.

Palabras claves: Arándano, Mercado, Estrategia de mercado, Exportación, Ventas.

\begin{abstract}
The blueberry fruit is an excellent source of vitamin C. There are also potential market segments in the United States that demand this fruit. Therefore, the present research entitled: MARKET STRATEGIES FOR THE EXPORT OF BLUEBERRIES (Vaccinium spp.), FROM THE ICA REGION, raises the research problem: What is the relationship that exists in the application of market strategies and the increase in exports of fresh Blueberries (Vaccinium spp.) from the Ica Region? Its objective is: To determine the relationship that exists in the application of market strategies and the increase in exports of fresh Blueberries (Vaccinium spp.) from the Ica region. It was started from the general hypothesis: The application of market strategies is related to the increase in exports of fresh Blueberries (Vaccinium spp.) From the Ica Region. An initial review of the biological description of the fruit, of the quality, of the exports, prices, commercialization and main national and international companies of production of this fruit was carried out. Likewise, an analysis of the different market strategies was carried out and it was determined that the market strategies that should be implemented by companies in the Ica region are: Communication and promotion marketing strategies, product strategy and concentric diversification strategy
\end{abstract}

Keywords: Blueberry, Market, Market Strategy, Export, Sales.

Como citar: García-Espinoza, A.J., Calderón-Huamani, D.F., Cabel-Moscoso, D.J. \& García-Espinoza, C. (2020). Estrategias de mercado para la exportación de arandano (Vaccinium corymbosum L.), de la región Ica. ÑAWPARISUN - Revista de Investigación Científica, 3(1), 71-82. 


\section{Introducción}

El arándano (Vaccinium corymbosum L.), es un cultivo frutal que ha experimentado en los últimos años un crecimiento sostenido en superficie y rendimiento en el Perú, convirtiéndose en una alternativa interesante de producción, principalmente en la costa norte del país donde se encuentra la mayor superficie de plantación (Agro negocios, 2014). El Perú cuenta con excelentes condiciones agroclimáticas, puede producir durante todo el año a lo largo de toda la costa y parte de la sierra debido a que presenta un gran potencial para producir arándanos de excelente calidad ya que de por sí se caracteriza por adaptarse fácilmente a cualquier entorno, por ello se debe de explotar el potencial con el que se cuenta (Minagri, 2016). El cultivo del arándano tiene un alto crecimiento en el sector frutícola pero, su cultivo comercial no está muy difundido en el departamento de lca. Se exporta a un mercado muy competitivo como Estados Unidos, esta realidad ha generado el interés de grandes empresarios a invertir en un fruto muy rentable. Por otro lado, el mercado de esta fruta se ha tornado más competitivo, tanto por el ingreso de nuevos países en el sistema productivo, como por la entrada en producción de nuevas áreas geográficas nacionales en arándano, una de las principales ventajas para la exportación, es la ubicación geográfica con respecto al mercado de destino (Estados Unidos), lo que permite el abastecimiento en contra estación (ventana comercial) obteniendo como consecuencia una elevada producción, un alza en precios y por ende un incremento en las exportaciones.

Asimismo, el desarrollo iqueño está basado en las exportaciones, es decir, en los beneficios derivados de la agroindustria. Actualmente las empresas productoras de arándanos ubicadas en Ica, Pisco y Chincha tienen hectáreas significativas de plantaciones de arándano y Estados Unidos es el principal consumidor de esta fruta y constituye un mega mercado con más de 275 millones de consumidores. Para competir en el mercado de EE.UU, es importante la calidad del producto producido, ya que la oferta es significativa, por lo tanto, se debe cumplir con las exigencias del mercado. Por lo que, para que lograr el éxito en la exportación de esta fruta, es necesario acompañarlo de estrategias de mercado que permita promocionarlo en el mercado internacional.

\section{Descripción biológica del Arándano:}

El arándano (Vaccinium corymbosum L.) es un arbusto frutal perteneciente a la familia de las Ericáceas, natural del este y medio oeste de Norteamérica, donde es denominado como Highbush blueberry o arbusto alto. El género Vaccinium presenta especies nativas en los Andes peruanos, tal como V. meridionale Sw., conocido como Gongapa o Agraz, ampliamente estudiada en Colombia. Géneros muy estudiados conjuntamente con V. corymbosum son V. ashei Reade, denominado Ojo de conejo (Rabbit-eye blueberry), y V. angustifolium (Aiton) Benth., denominado arbusto bajo (Lowbush blueberry) (Macbride, 1946; García \& García, 2006; Ávila et al, 2007). Según Francisco Caffarena (2010), son arbustos de tamaño mediano y hojas caedizas. Las raíces se encuentran principalmente hasta los 25 a $30 \mathrm{~cm}$ de profundidad. Más del 80 por ciento están sobre los $36 \mathrm{~cm}$ de profundidad y el 80 por ciento a $60 \mathrm{~cm}$ del centro de la corona (Carrera, 2012; Gough, 1980).

\section{Descripción del fruto del Arándano:}

El fruto es, botánicamente una baya. Color azul oscuro a negro. El tamaño es muy variable debido a las diferentes condiciones climáticas, varietales y de manejo que se presentan. Se reporta un rango de cuatro a $12 \mathrm{~mm}$ en ejemplares silvestres. Pulpa verde claro transparente, formada por el mesocarpio y endocarpio. Posee una cutícula cerosa de hasta $5 \mu \mathrm{m}$. El fruto contiene de una a varias semillas, de diez a 20, pero se ha reportado hasta 65. Madura en dos o tres meses, en función del ambiente y del cultivar. (Gough, 1994; Retamales \& Hancock, 2012; Floras, 2009). La composición química final aproximada del fruto es de 83 por ciento de agua, 15,3 de carbohidratos, 1,5 de fibra, 0,7 de proteína y 0,5 de grasas. El porcentaje de ácidos varía de uno a dos por ciento. (Retamales \& Hancock, 2012).

\section{Principales variedades o ecotipos de Arándano en el Perú}

Febres (2013) indica que según Federico Beltrán perteneciente a Fall Creek Genetics, la variedad que domina es Biloxi, explica que en el Perú hasta el 2011 no se había adherido a la UPOV (Unión Internacional para la Protección de Nuevas Variedades de Plantas); y que esto condicionó que el desarrollo inicial se haya llevado a cabo con variedades liberadas: Biloxi, Legacy, Misty, O’Neil, Duke, Brigitta, entre otras, siendo Beloxi la más exitosa en zonas bajas y desérticas de la costa que se estima abarcó entre el $80 \%$ y hasta el $90 \%$ de la superficie product iva en su momento y que en las alturas de la sierra se ha obtenido buenos resultados con Legacy. La Revista Agronegocios Perú (2014) explica que en el Perú existen variedades libres que se caracterizan por ser antiguas, con patentes vencidas, que nunca fueron protegidas o que son variedades desarrolladas por USDA; y variedades protegidas caracterizadas por tener acceso limitado y por tener que pagar un Royalty para su uso.

La primera plantación registrada en Perú estaba ubicada en Arequipa, en el año 2008, de 10 hectáreas. Hasta el 2009, el Perú no figuraba incluso como un productor no tradicional. (Aunque el país se encuentra en el octavo lugar entre los países exportadores de arándano, el crecimiento de las exportaciones durante el período 2012 - 2016 fue de 313 por ciento, el mayor de entre todos los países exportadores. (Bañados, 2009; MINAGRI, 2016; ITC, 2017).

La producción se reparte, al 2015 en 2500 las instaladas, en 7 regiones (Arequipa, Ancash, Cajamarca, Ica, La Libertad, Lambayeque, Lima); donde las unidades agrícolas mayores de 50 ha, poseen más de la mitad de las áreas. 
La Libertad es la región de mayor producción, ya que concentra casi el $90 \%$ de toda la producción nacional (Velásquez, 2014, MINAGRI, 2016). Al cierre del 2108 se contó con una exportación de 73776 toneladas lo cual significó un incremento del $72 \%$ en comparación al año 2017, año en el que se exportaron 42783 toneladas, con respecto a su valor FOB de exportación, el año pasado aumentó en 56\% en comparación al año 2017 (cuando alc anzó US\$ 356 millones) (AgroData Perú, 2018).

\section{Principales empresas peruanas exportadoras de Arándanos}

CAMPOSOL y TALSA, que representan el $88,0 \%$ del total exportado, el $20 \%$ corresponde a Ortifrutal, Blueberries Perú, Complejo Agroindustrial Beta, Agrícola Don Ricardo S.A.C, Agrícola La Venta, Danper Trujillo, entre otros. En lo que respecta a la comercialización, Rojas (2014) menciona que por ahora las únicas empresas que están exportando su producción y la de terceros son Camposol y Talsa ya que ambas cuentan con plantas de frío, sistema de transporte, logística y cuentan con mercado. Velásquez (2014) menciona que La Libertad, Áncash, Ica y Lima son las regiones que concentran las principales tierras de cultivo de arándano. Desde el año 2007, el departam ento de Ica constituye potencial para el cultivo de arándanos azules. Según el MINAGRI, se encontró que actualmente el departamento de Ica, tiene una participación del 7,8\% en el total de las exportaciones peruanas. MINAGRI, determinó que nuestro departamento cuenta con hectáreas que han sido cultivadas y se puede producir variedades High Bush y la variedad Biloxi.

\section{Estimaciones de la Demanda}

El mercado del arándano es uno de los más rentables del mundo y está dirigido a un público de alto poder adquisit ivo. (Arándanos, S.F.).Por otro lado, las técnicas y sistemas de cultivo que permiten que la producción de los países del hemisferio sur ingrese en contra estación está contribuyendo de forma positiva al crecimiento de la producción. (Guía del emprendedor, s. f.). Actualmente, el arándano es un fruto con altísima demanda, tal es así que en Europa Continental el consumo ha crecido más del $25 \%$ anual, Canadá ha mostrado incrementos en el consumo del $15 \%$, Estados Unidos $13 \%$ y en Asia el nivel de consumo ha tomado tanta fuerza que la producción se duplicó entre el 2012 y el 2014; mientras que en África fue casi el triple en el mismo periodo de tiempo. (León Carrasco, 2015). Vildósola (2007) señala que las estimaciones de la demanda están referidas a los análisis cualitativos y cuantitativos de las plazas donde se comercializan los productos dentro o fuera del mercado así como la codificación y decodificación de los factores inherentes al consumidor que generan percepción de calidad positiva a través de la satisfacción de necesidades.

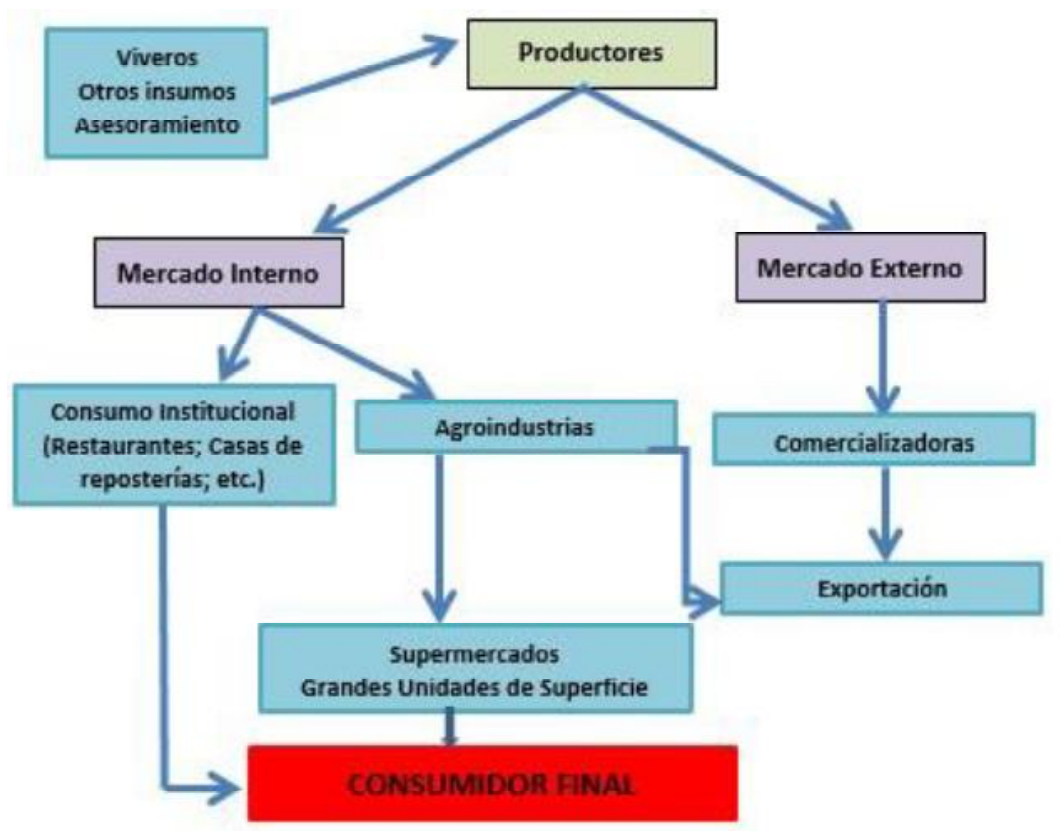

Figura 1. Cadena Comercial del Arándano 


\section{Estrategia de Marketing}

Manuera y Rodríguez (2007) menciona que las estrategias de marketing son los cursos de acción que mejor se adecúan a los recursos y capacidades y que han de permitirle a la organización a alcanzar la situación futura deseada.

\section{Estrategias alternas de Marketing}

\section{a. Penetración en nuevos mercados}

Cohen (2001) define cuatro tipos: La entrada (en un mercado nuevo se puede entrar el primero, pronto o tarde), la conquista del hueco (significa encontrar un segmento diferenciable del mercado, identificable por su tamaño, necesidades y objetivos y tratar de centrar todos los recursos en satisfacción de las necesidades de ese segmento), la ampliación (vertical la cual consiste en reunir dos o más etapas de la producción o de los procesos de comercialización; u horizontal que se refiere a la expansión de nuevos mercados) y el posicionamiento (se refiere a la posición que ocupa el producto en las mentes de los clientes en relación con los productos de la competencia).

\section{b. Expansión de la cuota de mercado}

Cohen (2001) define dos estrategias, la diferenciación o segmentación del mercado y la expansión general o limitada.

\section{c. Atrincheramiento}

Cohen (2001) señala que esta estrategia es necesaria cuando un producto se encuentra en la etapa de madurez o empieza ya a declinar, en cualquier caso el mercado ya no aumenta.

\section{Otras estrategias de marketing}

Sandhusen (2002) proporciona la siguiente clasificación de estrategias para el crecimiento del mercado:

\section{a..Estrategias de Crecimiento Intensivo}

Sandhusen (2002) menciona que consisten en "cultivar" de manera intensiva los mercados actuales de la compañía. Aplicadas en las oportunidades de "producto-mercado" existentes y que no han sido explotadas en su totalidad.

\section{b. Estrategias de Crecimiento Integrativo}

Sandhusen (2002) se refiere a que consiste en aprovechar la fortaleza que tiene una determinada compañía en su industria para ejercer control sobre los proveedores, distribuidores $\mathrm{y} / 0$ competidores.

\section{c. Estrategias de Crecimiento Diversificado}

Sandhusen (2002) señala que son adecuadas cuando hay pocas oportunidades de crecimiento en el mercado meta de la compañía.

\section{d. Retirada}

Cohen (2001) menciona que consiste en retirar el producto o servicio concreto del mercado, el único problema es cuándo y cómo, podemos destacar:

- Disminución de las ventas

- Obsolescencia tecnológica

- Moda

- Falta de competitividad

- Productos sustitutivos

\section{e. Estrategias de Liderazgo de Mercado o Estrategia de Líder}

Sandhusen (2002) menciona que son utilizadas por compañías que dominan en su mercado con productos superiores, eficacia competitiva o ambas cosas. Cutropia (2003) señala que con esta estrategia se llega a obtener mayores ingresos, lo que permitiría un incremento en la rentabilidad.

\section{f. Estrategias ofensivas}

Best (2007) señala que las estrategias ofensivas se orientan más al crecimiento y se dan especialmente en la fase de crecimiento del ciclo de vida de los productos.

\section{g. Estrategias de reto de mercado o estrategia del retador}

Sandhusen (2002) indica que son estrategias que las compañías pueden adoptar contra el líder de mercado y se clasifican en tres: Ataque frontal, el cual consiste en atacar toda la mezcla de mercado del líder; ataque en los costados, el cual consiste en enfocarse en los puntos débiles del líder, como el precio y las estrategias de derivación que consiste en enfocarse en áreas que no son abarcadas por el líder.

Cutropia (2003) menciona que el retador será una empresa que intenta expandir de una manera agresiva su cuota de mercado.

\section{h. Estrategias de seguimiento de mercado o estrategia del seguidor}

Sandhusen (2002) señala que son empleadas por las compañías de la competencia que no se interesan en retar al líder de manera directa o indirecta. Cutropia (2003) menciona que es la estrategia más sencilla pero también la más arriesgada; las empresas que siguen esta estrategia son aquellas que no pretenden hacer fuertes innovaciones ya que están de acuerdo con la cuota de mercado que tienen.

\section{i. Estrategias de nicho de mercado o estrategia del especialista}

Sandhusen (2002) menciona que son utilizadas por los competidores más pequeños porque están especializados en dar servicio a nichos del mercado 
y que los competidores más grandes suelen pasar por alto o desconocen su existencia. Cutropia (2003) señala que permite acceder a algunos nichos de mercado muy selectivos y en los que la competencia no suele ser muy fuerte.

\section{j. Estrategia basada en el ciclo de vida del producto}

Cutropia (2003) menciona que una vez analizadas las estrategias a seguir según Katler, sería interesante valorar en qué ciclo de vida se encuentra nuestro producto, esto permitirá centrar las tácticas posteriores.

\section{k. Estrategia F uncional: Marketing Mix - Las $4 \mathrm{P}$ del marketing}

Best (2007) señala que es conocido como estrategia de marketing táctico, lo que significa redactar estrategias en relación con el posicionamiento del producto, los precios, la comunicación y la distribución.

\section{Materiales y métodos}

Encuesta aplicada a las empresas exportadoras de arándanos frescos en la región Ica.

Se analizará la respuesta realizadas a las empresas exportadoras de arándanos frescos:

1. Ica: Agroindustrial Don Ricardo S.A.C. (20 ha. de Arándanos)

2. Ica: SUNFRUITS EXPORT S.A. (50 ha. de Arándanos)

3. Pisco-Humay: Agrícola Valle Pampa (45 ha de Arándanos)

4. Pisco: Frutos de Oro (20 ha. de Arándanos)

5. Chincha-Pueblo Nuevo: Agrícola Carmen Luisa S.A.C (30 ha de Arándanos.

\section{1. ¿ Su u empresa en que rubro está?}

\begin{tabular}{lr}
\hline \multicolumn{1}{c}{ Rubro } & Porcentaje (\%) \\
\hline Productos primarios & 55.0 \\
Productos procesados & 30.0 \\
Servicios & 10.0 \\
Otros & 5.0 \\
\hline Total & $\mathbf{1 0 0 . 0}$ \\
\hline
\end{tabular}

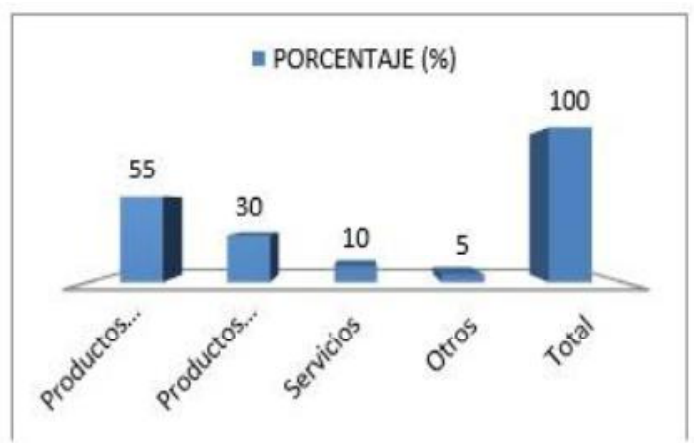

\section{Interpretación:}

El $55,0 \%$ de los entrevistados señalaron que el rubro de la empresa es productos primarios y el 30,0\% mencionó al rubro de productos procesados.

\section{2. ¿Pertenece a alguna asociación?}

\begin{tabular}{|c|c|}
\hline Asociación & Porcentaje (\%) \\
\hline Asociación de productores de arándanos & 35.0 \\
\hline Asociación de exportadores & 50.0 \\
\hline Ambos & 15.0 \\
\hline Total & 100.0 \\
\hline
\end{tabular}

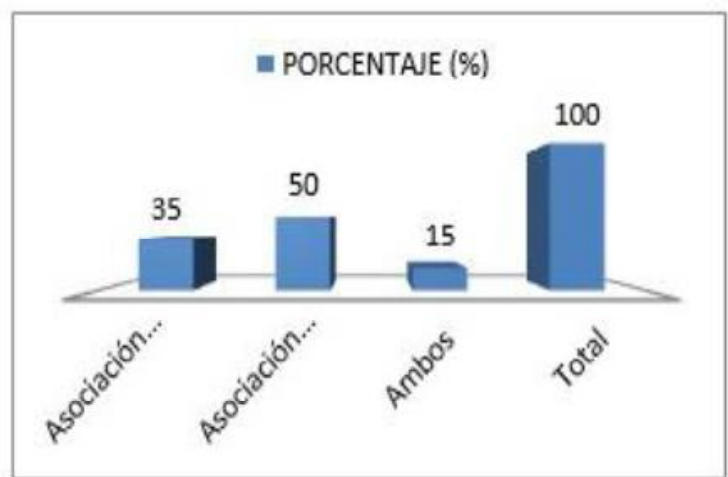

\section{Interpretación}

El 50,0 \% de los encuestados pertenecen a PROARANDANOS; el $35,0 \%$ pertenece a la Asociación de Exportadores y el 15,0 \% pertenece a ambos.

\section{3. ¿Conoce usted los atributos del arándano?}

\begin{tabular}{lrr}
\hline & Atributos & Porcentaje (\%) \\
\hline $\mathrm{Si}$ & & 98.0 \\
$\mathrm{No}$ & & 2.0 \\
\hline & Total & $\mathbf{1 0 0 . 0}$ \\
\hline
\end{tabular}

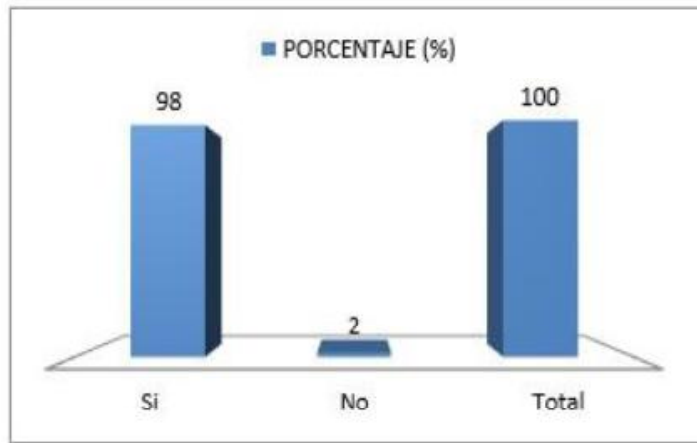

Interpretación

El 98,0\% de los encuestados indica que el principal atributo de este fruto es que es antioxidante y sus beneficios para la salud. 


\section{4. ¿Cuenta con áreas de cultivo de arándano?}

\begin{tabular}{lrr}
\hline & Áreas de cultivo & Porcentaje (\%) \\
\hline $\mathrm{Si}$ & & 89.0 \\
$\mathrm{No}$ & & 11.0 \\
\hline & Total & $\mathbf{1 0 0 . 0}$ \\
\hline
\end{tabular}

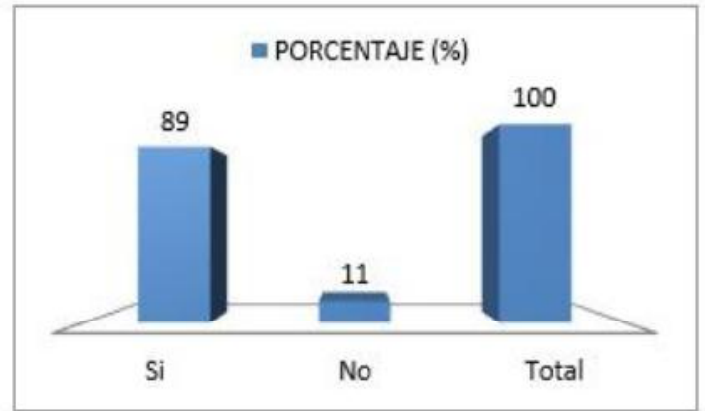

\section{Interpretación}

El $89,0 \%$ de los entrevistados indican que si tiene áreas de cultivo, el $11,0 \%$ no tiene áreas de cultivo.

\section{5. ¿Cuántas hectáreas de arándano tiene} sembrado?

\begin{tabular}{lr}
\hline \multicolumn{1}{c}{ Hectáreas (ha) } & Porcentaje (\%) \\
\hline Menor a una hectárea & 5.0 \\
Una hectárea & 10.0 \\
Dos hectáreas & 20.0 \\
Mayor a dos hectáreas & 65.0 \\
\hline Total & $\mathbf{1 0 0 . 0}$ \\
\hline
\end{tabular}

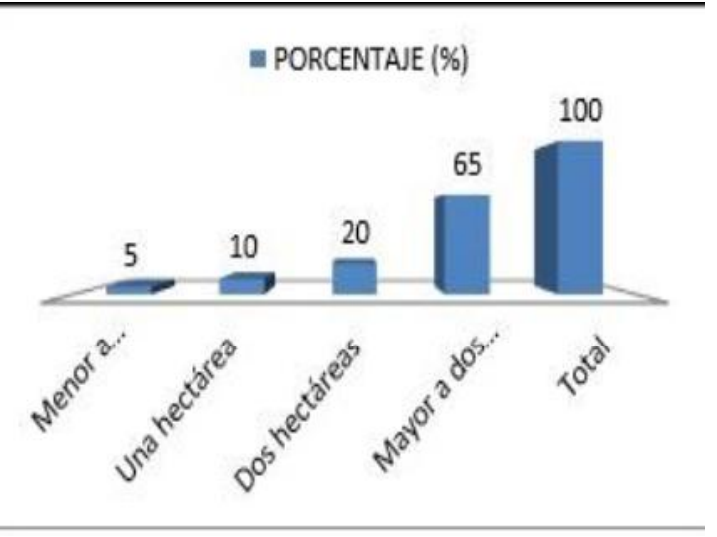

\section{Interpretación:}

El 65,0 de los entrevistados indicaron que tienen sembradas más de dos hectáreas. Actualmente, la técnica del cultivo en macetas es una alternativa para esta frutal porque el suelo en Perú no es completamente adecuado para este cultivo y otras de sus ventajas es que genera mayor productividad. Esta técnica se está consolidando en Ica.

\section{6. ¿Qué variedad de arándano tiene sembrado?}

\begin{tabular}{|c|c|}
\hline Variedad & Porcentaje (\%) \\
\hline Biloxi & 66.0 \\
\hline Misty & 18.0 \\
\hline Emeral & 10.0 \\
\hline Yugol & 6.0 \\
\hline Total & 100.0 \\
\hline
\end{tabular}

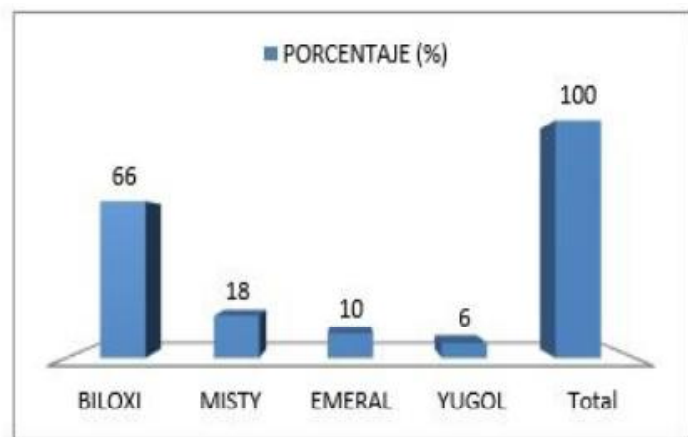

Interpretación:

El $66,0 \%$ señaló que siembra Biloxi; el 18,0\% señaló que siembra el Misty; el 10,0\% Emeral y el 6\% Yugol. También indicaron que una buena producción de arándanos empieza con la selección de una buena planta.

\section{7. ¿Por qué produce arándano?}

\begin{tabular}{lr}
\hline \multicolumn{1}{c}{ Producción } & Porcentaje (\%) \\
\hline Por calidad & 5.0 \\
Por precio & 50.0 \\
Por rendimiento & 5.0 \\
Oportunidad de mercado & 20.0 \\
Salida estacional & 20.0 \\
\hline Total & $\mathbf{1 0 0 . 0}$ \\
\hline
\end{tabular}

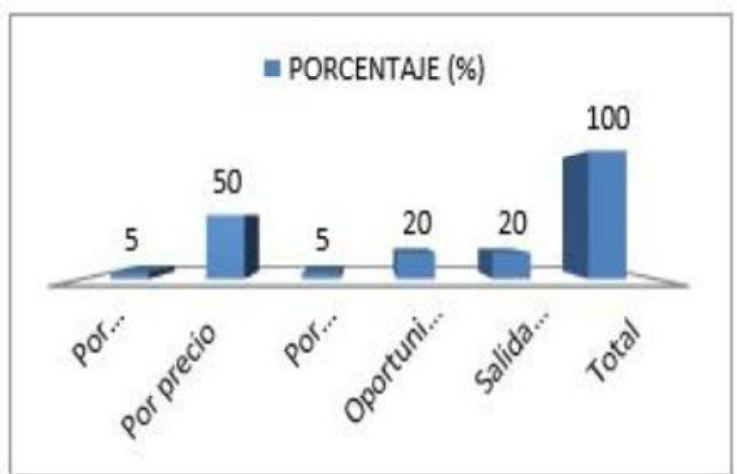

Interpretación:

El 50,0 \%, señalaron que producen arándanos por el precio que tiene en el mercado, el $20,0 \%$ por oportunidad y salida estacional. 


\section{8. ¿Conoce la cadena productiva del arándano?}

\begin{tabular}{lrr}
\hline & Cadena productiva & Porcentaje (\%) \\
\hline $\mathrm{Si}$ & & 80.0 \\
$\mathrm{No}$ & & 20.0 \\
\hline & Total & $\mathbf{1 0 0 . 0}$ \\
\hline
\end{tabular}

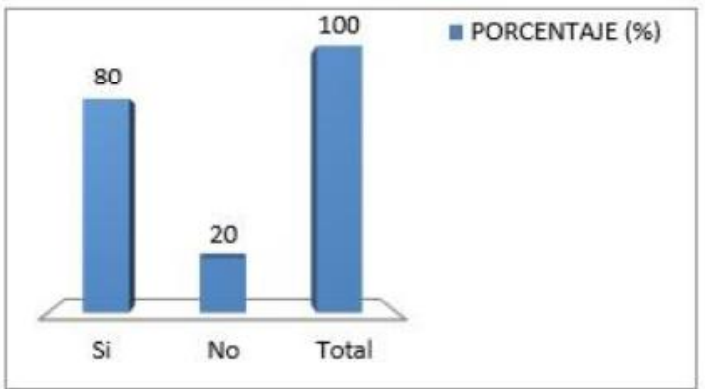

Interpretación

El 80,0\% indicó que Sí y el otro $20 \%$ indicó que NO.

\section{9. ¿De qué forma comercializa el arándano?}

\begin{tabular}{lr}
\hline \multicolumn{1}{c}{ Comercialización } & Porcentaje (\%) \\
\hline Fresco & 77.0 \\
Procesado (néctar, mermeladas, puré, & 13.0 \\
macerados & 10.0 \\
Otros & $\mathbf{1 0 0 . 0}$ \\
\hline Total & \\
\hline
\end{tabular}

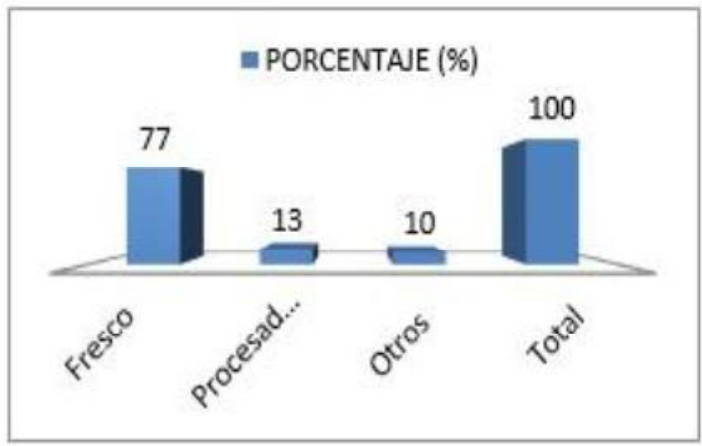

\section{Interpretación}

El 77\% indicó que lo comercializa fresco, el 13\% indicó que procesado.

10. ¿Cómo comercializa su producto, sea fresco o procesado?

\begin{tabular}{lr}
\hline \multicolumn{1}{c}{ Formas de comercialización } & Porcentaje (\%) \\
\hline Por pedidos & 23.0 \\
Mercado local & 17.0 \\
Mercado internacional & 60.0 \\
\hline Total & $\mathbf{1 0 0 . 0}$ \\
\hline
\end{tabular}

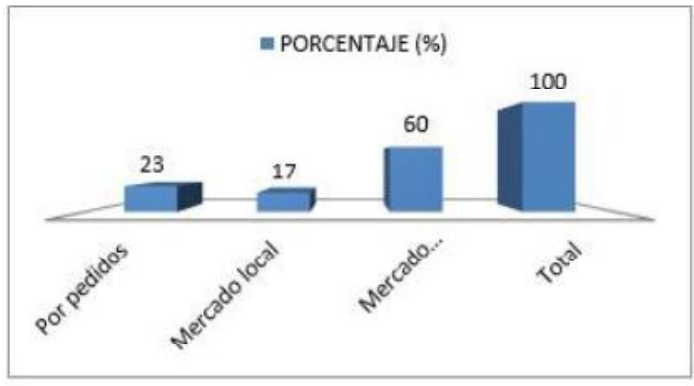

\section{Interpretación:}

El $60,0 \%$ los comercializa en el mercado internacional y el $23,0 \%$ por pedidos.

11. ¿Conoce la normativa para exportar el arándano fresco o procesado?

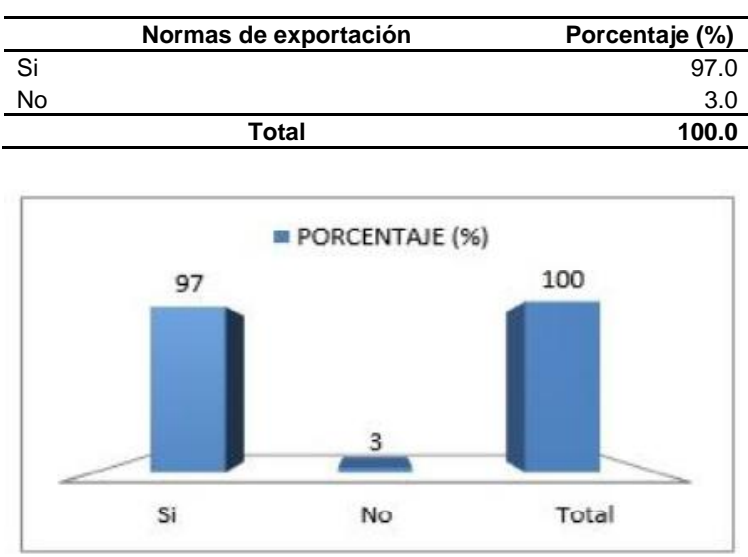

Interpretación:

El 97,0\% contestó que si conoce las normas para exportar, el $30 \%$ no conoce.

12. ¿Qué mecanismos de acceso al mercado ha utilizado?

\begin{tabular}{lr}
\hline \multicolumn{1}{c}{ Mecanismos } & $\begin{array}{c}\text { Porcentaje } \\
\text { (\%) }\end{array}$ \\
\hline $\begin{array}{l}\text { Combinación venta directa y venta Trading } \\
\text { Companies }\end{array}$ & 40.0 \\
Combinacion de venta a minoristas y clientes & 20.0 \\
nacionales exportadores & 20.0 \\
Licencias o franquicias \\
$\begin{array}{l}\text { Venta a intermediarios internacionales } \\
\text { Venta a clientes nacionales que luego exportan el } \\
\text { producto }\end{array}$ \\
\hline Total & 10.0 \\
\hline &
\end{tabular}




\section{Interpretación}

El 40,0\% contestó que es mediante una combinación de venta directa y venta a tradings companies. Esta modalidad de venta tiene ventajas para los distribuidores, como para el consumidor final, que se han convertido en clientes de los productores. El $20,0 \%$ lo hacen mediante combinación directa.

13. ¿Conoce los criterios empleados para establecer los precios de exportación?

\begin{tabular}{lr}
\hline \multicolumn{1}{c}{$\begin{array}{c}\text { Criterios para fijación de precios de } \\
\text { exportación }\end{array}$} & Porcentaje (\%) \\
\hline Costo & 12.0 \\
Competencia & 8.0 \\
Elasticidad de la demanda & 47.0 \\
Yo difino el precio & 3.0 \\
Lo propone el comprador & 30.0 \\
\hline Total & $\mathbf{1 0 0 . 0}$ \\
\hline
\end{tabular}

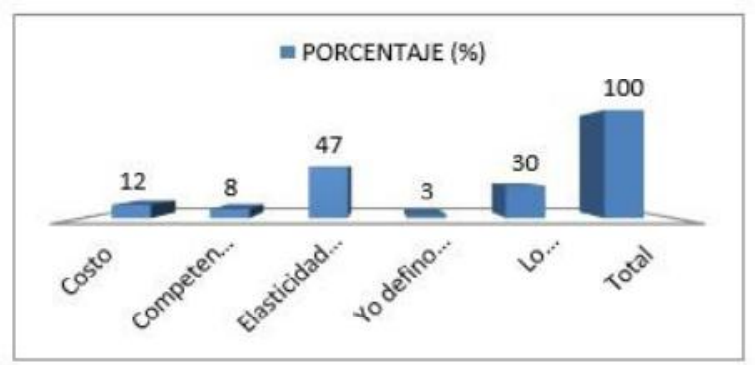

\section{Interpretación}

El 47,0\% señaló que está en función a la elasticidad de la demanda, el 30,0\% indicó que lo propone el comprador y el $12,0 \%$ señaló que se basa en el costo.

\section{4. ¿Su producto tiene alguna marca?}

\begin{tabular}{lrr}
\hline & Normas de exportación & Porcentaje (\%) \\
\hline $\mathrm{Si}$ & & 72.5 \\
$\mathrm{No}$ & & 27.5 \\
\hline & Total & $\mathbf{1 0 0 . 0}$ \\
\hline
\end{tabular}

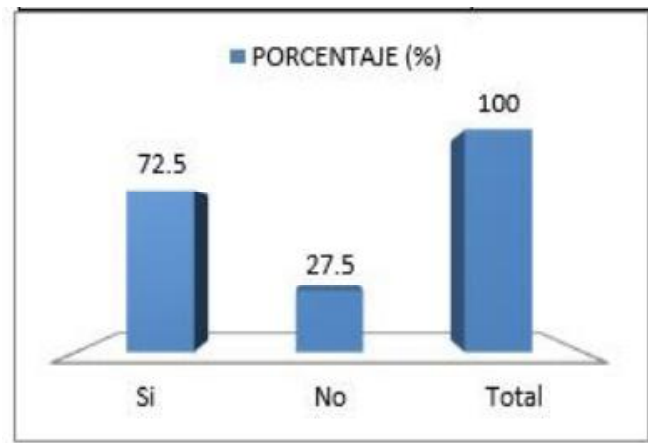

\section{Interpretación}

El $72,5 \%$ indicó que si tiene marca de exportación, mientras que el $27,5 \%$ señaló que no tiene marca.

\section{5. ¿Cuál es su modalidad de exportación?}

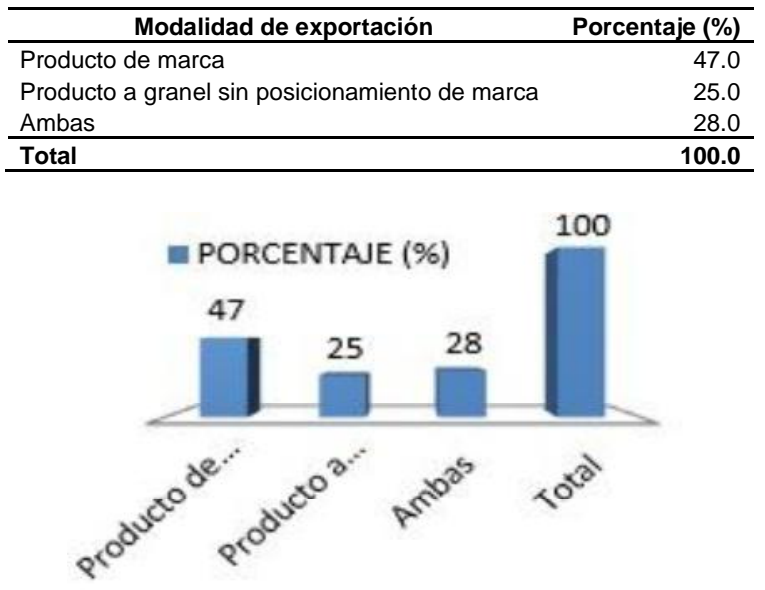

\section{Interpretación}

El $47,0 \%$ indica que exporta sus productos con marca, el $25,0 \%$ exporta como productos a granel y el 28,0 \% señaló que con ambas modalidades. La ventaja de tener productos con marca, garantiza posicionar a nivel internacional, la calidad, la variedad y los beneficios de la oferta de arándanos.

16. ¿La empresa tiene norma de certificación Internacional?

\begin{tabular}{lrr}
\hline & Normas de certificación internacional & Porcentaje (\%) \\
\hline $\mathrm{Si}$ & & 97.0 \\
$\mathrm{No}$ & 3.0 \\
\hline & Total & $\mathbf{1 0 0 . 0}$ \\
\hline
\end{tabular}

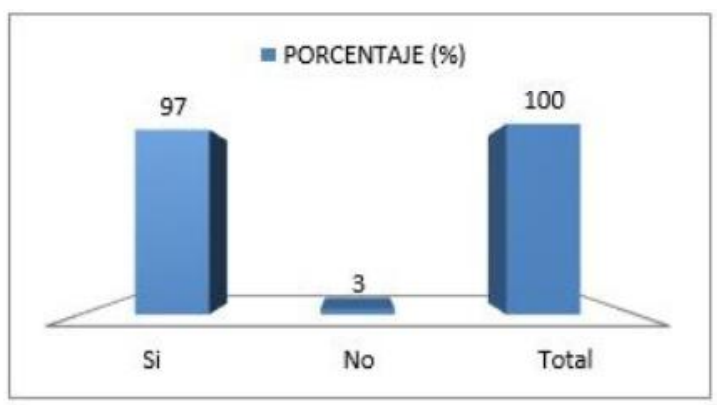

Interpretación

El 97,0\% contestó que si cuenta con la certificación GLOBALG.A.P y el 3\% no tiene.

\section{7. ¿Es parte de un consorcio de exportación?}

\begin{tabular}{lrr}
\hline & Consorcio de exportación & Porcentaje (\%) \\
\hline $\mathrm{Si}$ & & 72.0 \\
$\mathrm{No}$ & Total & 28.0 \\
\hline & & $\mathbf{1 0 0 . 0}$ \\
\hline
\end{tabular}




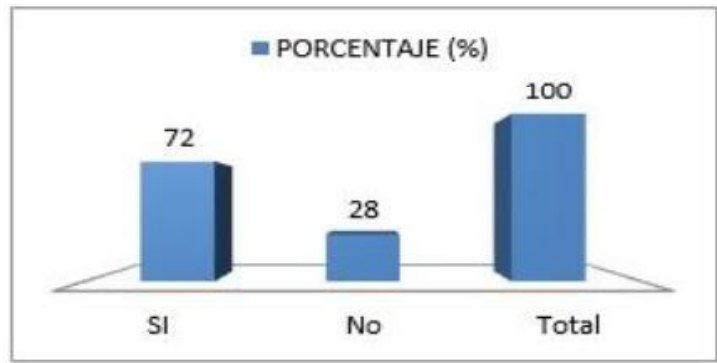

\section{Interpretación}

El $72,0 \%$ contestó que si pertenecen algunos a PROCHINCHA, PROARANDANOS, ADEX; y el $28,0 \%$ contestó que NO.

\section{8. ¿De qué manera promociona su producto?}

\begin{tabular}{lr}
\hline \multicolumn{1}{c}{ Promoción del producto } & Porcentaje (\%) \\
\hline Mediante ferias internacionales & 50.0 \\
Mediante el Estado & 5.0 \\
Por internet & 25.0 \\
Mediante revistas del área & 15.0 \\
No promocionan & 5.0 \\
\hline Total & $\mathbf{1 0 0 . 0}$ \\
\hline
\end{tabular}

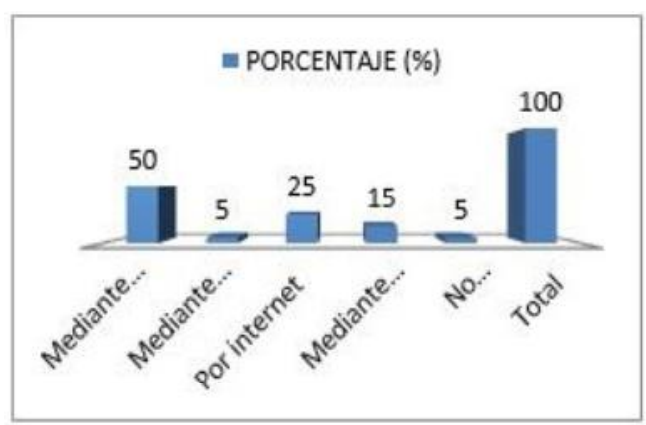

\section{Interpretación}

El $50,0 \%$ señala que promociona sus productos en ferias internacionales; el 25,0\% mediante internet y el $15,0 \%$ mediante revistas, especializadas. La ventaja de participar en ferias permitirá a los productores:

- Promocionar sus productos

- Contactar compradores y proveedores

- Conocer los productos de las empresas competidoras

- Conocer las tendencias del mercado, nuevos canales de distribución, segmentos del mercado, entre otros.

\section{9. ¿Qué atributos diferencian sus productos?}

\begin{tabular}{lr}
\hline \multicolumn{1}{c}{ Atributos del producto } & Porcentaje (\%) \\
\hline Calidad & 54.0 \\
Precio & 18.0 \\
Envase & 5.0 \\
Etiquetado & 3.0 \\
Atributos sensoriales & 10.0 \\
Oportunidad & 10.0 \\
\hline Total & $\mathbf{1 0 0 . 0}$ \\
\hline
\end{tabular}

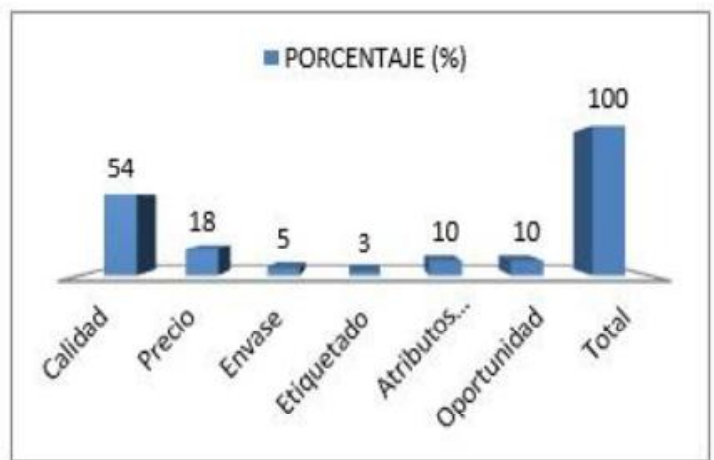

\section{Interpretación}

El $54,0 \%$ de los encuestados mencionó que la calidad, el $18,0 \%$ mencionó precio y el $10,0 \%$, indicó los atributos sensoriales (dulce) y la oportunidad de mercado. Por su calidad y precio el arándano peruano está en crecimiento de 494,5\%, según el Departamento de Comercio de Estados Unidos, durante los últimos cinco años y habría empezado a desplazar a los otros países exportadores (Chile), aquellos que en algún momento dominaron el mercado.

\section{0. ¿Qué carencias considera le limita exportar} arándanos?

\begin{tabular}{lr}
\hline \multicolumn{1}{c}{ Carencias para exportación } & $\begin{array}{c}\text { Porcentaje } \\
\text { (\%) }\end{array}$ \\
\hline Escasez de productos agrícolas & 6.0 \\
Escasez de conocimientos del manejo del & 19.0 \\
producto & 20.0 \\
Escasa tecnología de procesamiento & 10.0 \\
Falta de logística adecuada & 10.0 \\
Falta de cadena de frío & \\
Falta de financiamiento a pequeños y medianos & 30.0 \\
productores & 5.0 \\
Falta de espacios para áreas de cultivo & $\mathbf{1 0 0 . 0}$ \\
\hline Total & \\
\hline
\end{tabular}

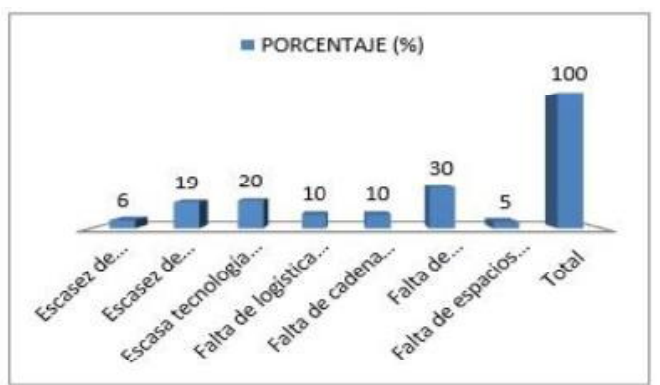

\section{Interpretación}

Las respuestas fueron variadas, el $30,0 \%$ señalo que falta financiamiento a pequeños y medianos productores, el $20,0 \%$ escasa tecnología en el procesamiento y el $19,0 \%$ en el manejo del producto. 


\section{Resultados y discusión}

\section{Propuesta de Estrategias de Mercado}

\section{Estrategias ofensivas}

Estrategia Ofensiva Básica l: Invertir para Aumentar las Ventas en mercados.

\section{Estrategia Ofensiva IA: Crecimiento de la cuota de mercado}

Se debe determinar el Índice de desarrollo de la cuota de participación.

Cuota de mercado real del año $2018=5,2 \%$

Para hallar la cuota de mercado potencial, se estimó el incremento de la cuota que Perú puede tener con la práctica de una estrategia de Marketing táctico, donde:

- $80 \%$ de notoriedad

- $80 \%$ de preferencia

- $80 \%$ de intención de compra

- $70 \%$ de disponibilidad

- $75 \%$ de nivel de recompra

\section{Estrategias que influyen en la cuota de mercado}

\section{A.1. Estrategia de Comunicación y Promoción: Notoriedad del producto}

\section{Objetivos:}

1. Resaltar el producto en el mercado objetivo.

2. Buscar que el consumidor conozca el producto y sus beneficios.

Táctica:

Publicitar en revistas especializadas, internet, ferias y promociones de ventas. Las estrategias serían:

- Promover alianzas estratégicas con operadores internacionales.

- Diseño de páginas web, para publicitar los beneficios para la salud del arándano.

- Empleo del E-mail marketing que tiene un costo muy reducido.

- Participación en diferentes eventos nacionales e internacionales.

- Publicación en revistas nacionales e internacionales.

A.2. Estrategia de Producto: Atractivo del Producto (Preferencia)

\section{Objetivos:}

Hacer que el producto resulte atractivo para el consumidor.

\section{Tácticas:}

- Producto: Arándano fresco y procesado

\section{Calidad:}

Como arándano fresco (materia prima): Color azul uniforme, de textura compacta, tamaño uniforme (calibre solicitado por el cliente), con $11^{\circ}$ Brix.

Como arándano procesado: mermeladas, pulpas, jaleas, licores, refrescos, etc.

$\checkmark \quad$ Marca: El $37 \%$ de los exportadores no tienen una marca de comercialización, es necesario que lo tengan para posicionarse en el mercado objetivo.

$\checkmark$ Diseño: El exportador debe tener productos alternativos como arándano congelado, jugos, jaleas, mermeladas, compotas, etc.

$\checkmark \quad$ Envase y embalaje:

Como arándano fresco:

Envase: En CLAMSHELLS de 6 oz, 12 oz, 18 oz y 4,4 oz; y en CLAMSHELLS de 4,4. oz.

$\checkmark \quad$ Embalaje: Caja de cartón de1,5; 2,0; 3,7; 4,0; 3 $\mathrm{kg}$

$\checkmark \quad$ Etiqueta: debe de tener la DENOMINACIÓN de la fruta, contenido neto $\mathrm{y}$ datos legales del productor, envasador e importador

$\checkmark$ Ciclo de vida del producto: El fruto en el mercado de EE.UU está en la etapa de CRECIMIENTO, por lo tanto, las ventas aumentarían.

\section{A.3. Estrategia de Precio}

\section{Objetivos:}

Propiciar la compra de los potenciales clientes.

\section{Táctica:}

- Analizar la estructura de costos de producción.

- Aprovechar la ventana comercial del mercado de EE.UU., en los meses de Agosto, Setiembre y Octubre, donde el precio de venta es de 20 dólares el kilogramo, y en meses de enero a febrero es de 4 y 5 dólares el kilogramo.

\section{A4. Estrategias de Plaza - Distribución}

\section{Objetivos:}

El fruto debe tener una alta disponibilidad, es decir, presencia en el punto de venta.

\section{Tácticas:}

- Definir el canal de distribución:

$\checkmark$ Fabricante - Consumidor final

$\checkmark$ Fabricante-Minorista-Consumidor final 
$\checkmark \quad$ Fabricante-Mayorista-MinoristaConsumidor final

$\checkmark$ Fabricante-Agente-Mayorista-MinoristaConsumidor final.

- El medio de transporte (aéreo o marítimo) en función al mes a exportar.

- En el canal de distribución directo a los minoristas, establecer exhibidores.

- Diseñar una página web o una tienda virtual, para impulsar la disponibilidad del producto.

\section{A.5. Estrategia de experiencia del cliente}

Objetivos: Los productos a comercializar deben tener un buen servicio en la compra.

\section{Tácticas:}

$\checkmark$ Tener una persona ya sea presencial o virtual para la atención.

$\checkmark$ Establecer protocolos de atención, para los clientes.

\section{Estrategia Ofensiva IB: Aumenta los ingresos} por cliente

\section{A. Estrategia de Diversificación Concéntrica (Relacionada)}

Objetivo: Extender la línea de productos para aumentar el nivel de consumo de cada cliente y lograr ingresar a nuevos mercados.

Táctica: Impulsar exportaciones de confituras jaleas, mermeladas, purés y pastas de frutas que se han incrementado, por lo tanto, se debe diversificar los productos derivados de arándano.

B. Estrategia de Precios Superiores "Price Premium"

Objetivo: Adicionar a los productos con nuevos servicios o con calidad superior.

Táctica: Exportar el fruto con la calidad que exige el mercado, que determina que los consumidores lo adquieran y se puede utilizar la estrategia de Price Premium.

\section{Estrategia Ofensiva IC: Entrada en Nuevos} Segmentos de Mercado

Objetivo: Incrementar las ventas en base a la estrategia de crecimiento de desarrollo de nuevos mercados con los productos actuales.

Táctica: Implementar la venta por canal online, donde el consumidor encuentre precios bajos, lo que generaría demanda del consumidor.

\section{d. Estrategia ofensiva ID: Expandir la Demanda Global del Mercado}

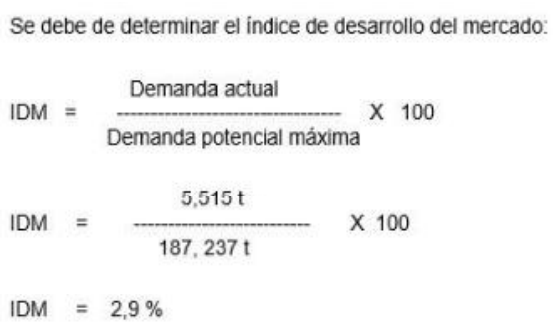

El valor de 2,9\% determina que existe un crecimiento en el mercado.

III. Estrategia Ofensiva IIC: Disminución de costes/Mejora de la Productividad de Marketing

Objetivo: Obtener márgenes de ganancia rentables.

\section{Táctica:}

- Analizar la reducción de costos.

- Evaluar la estructura de costos (mano de obra para cosecha y empacado)

- Uso de equipo de clasificación para el envasado.

\section{d. Estrategia ofensiva IID: Desarrollo de Ventajas} en Marketing

Objetivo: Incrementar las ventas a través del Marketing.

Táctica: Aplicar una marca de calidad.

\section{Estrategia Ofensiva Básica III: Entrada a Nuevos Mercados:}

1. Entrada a nuevos mercados similares.

2. Entrada a nuevos mercados no relacionado

3. Entrada a nuevos mercados emergentes

4. Desarrollo de nuevos mercado

\section{a. Estrategia Ofensiva IIIA: Entrada en Nuevos Mercados Relacionados}

Objetivo: Identificar nuevas fuentes de crecimiento para aumentar la participación del arándano en el mercado americano.

Táctica: Trabajar con otras variedades de berries.

- Sumarle un valor agregado a los arándanos. 


\section{Conclusiones}

Las estrategias de mercado que deben ser implementadas son: Estrategias de marketing de comunicación y promoción, estrategia del producto, estrategia de diversificación concéntrica, estrategia de mejora de la lealtad y el nivel de recompensa, estrategia de mejora de la ventaja de diferenciación. Por lo tanto, existe una relación directa en la aplicación de estrategias de desarrollo de mercado que derivaría en el incremento de las exportaciones de Arándano fresco a Estados Unidos.

Las características del mercado americano en relación a la regulación alimentaria es exigente, el consumidor adquiere esta fruta durante todo el año y exige calidad. Asimismo, se identificó que si invierte en la mejora de la producción de Arándano fresco, la ventana comercial pasaría ser desde setiembre a diciembre.

Se ha identificado que el mercado de Estados Unidos, es potencial consumidor de Arándano fresco por sus propiedades nutricionales y medicinales y tiene la economía más grande del mundo. Por lo que la estrategia ofensiva identificada (producto, precio, distribución y comunicación), permitiría incrementar las ventas a este mercado.

Las ventas de las exportaciones de este fruto se incrementaron en $70,0 \%$ en un promedio de diez años, aplicando estrategias de mercado se puede incrementar más las ventas. Asimismo, el nivel de posicionamiento del arándano en el mercado de Estados Unidos es óptimo, porque está ocupando el octavo lugar entre las veinte frutas que el consumidor prefiere y tiene demanda en todas las edades y estratos sociales.

\section{Recomendaciones}

- El Instituto Nacional de Calidad (INACAL), debe de elaborar una norma técnica para que el arándano de nuestro país cumpla con los requisitos de la demanda de mercado internacional.

- Sierra y Selva Exportadora conjuntamente con el Ministerio de Agricultura y Riego, deben desarrollar 0 implementar programas de capacitación en la cosecha, post cosecha, procesamiento y comercialización del arándano, con la finalidad de mejorar su rentabilidad.

- Realizar investigaciones relacionadas a variedades de arándanos que se adapten a los factores ambientales o zonas de producción, para optimizar su rendimiento y calidad.

- Posicionar al Arándano fresco, en ferias especializadas más importantes del sector de Estados Unidos, que permita exhibir el producto y generar contactos con empresas y conocer las nuevas tendencias del sector, analizar la competencia de otros productores de esta fruta.

- Las empresas exportadoras de arándanos deben de aplicar las estrategias de mercado propuestas en el capítulo de conclusiones del presente estudio.
- Identificar a los productores del arándano del departamento de Ica y del Perú a través de un censo agrario, con la finalidad de estimar la cantidad de hectáreas cultivables y la oferta exportable de este fruto.

\section{Referencias bibliográficas}

Barichivich, J. (2010). El canal de distribución del arándano en fresco exportado desde Chile a los Estados Unidos. Tesis para optar al grado de Licenciado en Agronomía. Chile: Universidad Austral de Chile.

Benavides, L. (2014). Programa nacional de berries. Lima: Sierra exportadora.

Benites, L., Ruff, C. (2014). Ingeniería económica: Aplicada a las decisiones de inversión y financiación de la empresa ( $2^{a}$ ed.). Trujillo, Perú: Compañía Editorial Americana S.R.L.

Daniels, J., Rudabaugh, L.; Sullivan, D. (2009). Negocios internacionales. (12 ${ }^{\mathrm{a}}$ ed.). México: Editorial Pearson.

Fred, R. (2013). Conceptos de administración estratégica (14aㅡ ed.). México: Editorial Pearson.

Hernández, R., Fernández, C., Baptista, M. (2010). Metodología de la investigación. ( $5^{\mathrm{a}}$ ed.). México: Editorial McGraw-Hill.

Leyton, M., \& Rodríguez, A. (2009). Prospección y Exportación de arándanos frescos al mercado Estado Unidense. Tesis para optar al título de ingeniero comercial. Chile: Universidad de Talca

Mateo, J. (2005). Prontuario de agricultura (1 ${ }^{\underline{a}}$ ed.) Madrid, España: Grupo Mundi-Prensa.

Medina, M., \& SÁNCHEZ, M. (2016). Producción y exportación de arándanos para Estados Unidos. Tesis para optar el grado académico de magister en Administración de Empresas. Lima: UPC.

Paulini, N., \& Viguria, J. (2016). Propuesta para incrementar la oferta exportable peruana mediante la exportación de arándanos a los Estados Unidos de Norteamérica. Tesis para optar el título de Licenciada en Administración y Negocios Internacionales. Lima: UPNW.

Radebaugh, L., Suvillan, D. (2009). Negocios internacionales (12 ${ }^{\mathrm{a}} \mathrm{ed}$,). México: Editorial Pearson.

Ruiz, A. (2007). Manual de derecho mercantil (1 ${ }^{\mathrm{a}}$. ed). Madrid, España: Universidad Pontificia Comillas.

Salazar, K. (2014). Oportunidades de negocio en el mercado de Estados Unidos para las exportaciones de arándanos frescos provenientes de la región La Libertad. Tesis para optar el grado de licenciado en administración de negocios internacionales. Perú: Universidad Privada del Norte.

Villa, C. (2013). Proyecto de factibilidad para producción y exportación de jugo natural de arándano al mercado árabe. Tesis para optar el grado de ingeniero en comercio exterior y negociación internacional. Escuela Politécnica del Ejército, Ecuador. 